\title{
El estilo narrativo en H.P. Lovecraft: un análisis cuantitativo de The Call of Cthulhu y otras obras icónicas
}

Narrative style in H.P. Lovecraft: a quantitative analysis of The Call of Cthulhu and other iconic tales

JOSÉ LUIS ARROYO BARRIGÜETE

UNIVERSIDAD PONTIFICIA COMILLAS

ORCID: https://orcid.org/0000-0002-3660-3933

RESUMEN: Este trabajo analiza la presencia de un patrón muy específico tanto en The Call of Cthulhu como en otras de las obras icónicas de Lovecraft. Combinando el análisis literario con técnicas computacionales de minería de textos, se ha podido cuantificar dicho patrón, que se articula alrededor de tres ejes. En primer lugar, la creación de una atmósfera vertebrada en torno a los sentimientos de miedo, confianza y tristeza, que además se presentan exactamente en ese orden de importancia, y cuya combinación genera emociones de sumisión y desesperación. El segundo eje está relacionado con el sentimiento de confianza, concentrada principalmente al principio de la obra, momento en el que se busca precisamente dotar de credibilidad a los extraordinarios hechos que se relatarán más adelante, en el clímax de la obra. Por último, el tercer eje es el giro final, en el que se produce una reacción de quien entra en contacto con las monstruosas criaturas, alcanzando una pírrica victoria. Un sentimiento de ira creciente a lo largo de toda la obra, que alcanza su máximo precisamente en este último evento, refuerza esta idea de reacción última y desesperada.

Palabras clave: Lovecraft, La llamada de Cthulhu, minería de textos, análisis de sentimientos, estilística.

ABSTRACT: This paper analyzes the presence of a very specific pattern in The Call of Cthulhu as well as in other of Lovecraft's iconic tales. By combining literary analysis with computational text mining techniques, it has been possible to quantify this pattern, which is articulated around three axes. Firstly, the creation of an atmosphere that is structured around feelings of fear, trust and 
sadness, which are also presented in exactly that order of importance, and whose combination generates emotions of submission and despair. The second axis is related to the feeling of trust, that is mainly concentrated at the beginning of the tale, a moment in which it is sought precisely to give credibility to the extraordinary events that will be recounted later, at the climax. Finally, the third axis is the final twist, in which there is a reaction from those who come into contact with the monstrous creatures, achieving a pyrrhic victory. A growing feeling of anger throughout the tale, which reaches its peak precisely in this last event, reinforces this idea of ultimate and desperate reaction.

Key words: The Call of Cthulhu, text mining, sentiment analysis, stylistic.

\section{INTRODUCCIÓN}

La obra de Lovecraft ha tenido no solo un enorme impacto en la cultura popular, inspirando desde películas ${ }^{1}$ y comics, hasta música ${ }^{2}$ y videojuegos (Smith, 2016), sino también en otros ámbitos de los más inesperado: por sorprendente que parezca, dado el marcado carácter racionalista y ateo de Lovecraft, la mitología que creó ha ejercido y sigue ejerciendo influencia en prácticas ocultistas (Steadman, 2015). Del mismo modo, tanto su obra como la propia figura de Lovecraft, han despertado gran interés entre la comunidad académica, dando lugar a investigaciones de lo más diverso, e incluso a la existencia de una revista académica, Lovecraft Annual ${ }^{3}$, dedicada a su estudio.

Muchos de los relatos de Lovecraft se articulan en torno a una mitología propia, no del todo coherente (De Camp, 2011), que pone el foco en la insignificancia de la raza humana (Harman, 2010). Como señala Houellebecq en relación a Lovecraft «Few beings have ever been so impregnated, pierced to the core, by the conviction of the absolute futility of human aspiration [...]. Everything will disappear. And human actions are as free and as stripped of meaning as the unfettered movement of the elementary particles»» (2019: 40-41). Ligado a esto, en los relatos de Lovecraft la humanidad se encuentra rodeada por misterios y criaturas tan poderosas que escapan no ya de su control, sino de su mera comprensión, reforzando precisamente esta idea de insignificancia. El problema, desde un punto de vista literario, consiste precisamente en cómo «depict with mere words» lo inenarrable (Mariconda, 1991: 189), y como señala Stableford (2007: 71) «the entire tradition of cosmic horror fiction can be regarded as a heroic but doomed attempt to rise to that challenge: to communicate the uncommunicable».

En el conjunto de la obra de Lovecraft, The Call of Cthulhu constituye un hito clave, no solo porque «it was only in The Call of Cthulhu (1926) that the Cthulhu Mythos, as such, can (retroactively) be said to have come into genuine existence» (Joshi, 2007: 102), sino también porque se trata de «one of the central documents of Lovecraft's

\footnotetext{
${ }^{1}$ Aunque como apunta Menegaldo (2019: 78) «cinema rarely manages to convey a sense of Lovecraft's materialistic, relativistic worldview».

${ }^{2}$ De hecho, la influencia de Lovecraft en la música, y en particular en diferentes subgéneros del heavy metal, es especialmente relevante, pues como señala Peregrina Castaños (2019: 101), «A diferencia de lo que sucede con muchos otros productos culturales, en esta música, y en especial en sus modalidades extremas, sí se ha conseguido plasmar [la cosmogonía de Lovecraft]».

${ }^{3}$ Que a su vez fue precedida por Lovecraft Studies (1980-2005), revista con el mismo foco temático.
} 
aesthetic of the weird» (Joshi, 2014c). De hecho, el texto con el que se inicia este relato es, probablemente, el mejor resumen de la cosmovisión que transmiten sus obras:

The most merciful thing in the world, I think, is the inability of the human mind to correlate all its contents. We live on a placid island of ignorance in the midst of black seas of infinity, and it was not meant that we should voyage far. The sciences, each straining in its own direction, have hitherto harmed us little; but some day the piecing together of dissociated knowledge will open up such terrifying vistas of reality, and of our frightful position therein, that we shall either go mad from the revelation or flee from the light into the peace and safety of a new dark age

(Lovecraft, 2017a[1928]: 36)

Desde una perspectiva estilística, resulta de interés analizar hasta qué punto el escritor logra provocar el efecto deseado, que en este caso y partiendo de una atmósfera de oscuridad y melancolía (Norman, 2013), busca una combinación de sentimientos de insignificancia, desesperación y miedo. Este es precisamente el objetivo del presente trabajo, cuya hipótesis central es que The Call of Cthulhu, al igual que otras de sus obras, se articula en torno a tres ejes íntimamente ligados. En primer lugar, la creación de una atmósfera muy concreta vertebrada alrededor de las emociones de miedo, confianza y tristeza, que además se presentan en ese orden de importancia. Es la combinación de estas emociones la que genera sumisión y desesperación, elementos clave en la filosofía propia del horror cósmico. El segundo eje está relacionado con el sentimiento de confianza, que se concentra principalmente al principio de la obra, momento en el que se busca dotar de credibilidad a los extraordinarios hechos que se relatarán más adelante, especialmente al final de la obra. Por último, el tercer eje es el giro final, en el que la reacción de quien entra en contacto con las monstruosas criaturas le confiere una pírrica victoria. Un sentimiento de ira creciente a lo largo de toda la obra, que alcanza su máximo precisamente en este último evento, refuerza esta idea de reacción última y desesperada, de quien se revela a su fatal destino pese a ser consciente de la futilidad de sus esfuerzos. Adicionalmente, en el caso de The Call of Cthulhu, al igual que sucede en alguna otra obra, este clímax se ve reforzado por un súbito cambio en el cronotopo.

A fin de verificar esta hipótesis, el presente trabajo se estructura en tres partes diferentes. En la primera se detallan los aspectos metodológicos de la investigación. A continuación, se lleva a cabo un análisis de The Call of Cthulhu, incidiendo en los aspectos estilísticos que contribuyen a conformar el hilo narrativo de la obra, y complementando el análisis mediante el uso de herramientas analíticas de minería de textos. Por último, se analiza cómo este mismo patrón aparece en otras de sus obras, lo que nos lleva a concluir que, efectivamente, Lovecraft utiliza este esquema de una forma intencionada y recurrente.

\section{ASPECTOS METODOLÓGICOS}

Tomando en consideración el objetivo mencionado, el estudio necesita ser abordado desde la interconexión de tres áreas diferentes: el análisis literario, la psicología de las emociones y, a fin de poder cuantificar el análisis, el uso de herramientas computacionales de minería de textos (text mining). En primer lugar, solo desde el análisis literario se pueden captar determinados elementos clave de la obra, imposibles de identificar mediante el mero estudio cuantitativo. Este es el caso, por ejemplo, del uso que hace Lovecraft de diferentes cronotopos (Bakhtin, 1990) a lo largo de la obra. Como ya se ha mencionado, el tercer eje del análisis que se pretende desarrollar es un giro final, ligado al contacto con terribles criaturas, que en dos de las cuatro obras analizadas se refuerza con un súbito cambio en el cronotopo. Es decir, Lovecraft acompaña el patrón 
emocional del relato con una alteración de los elementos espacio temporales, alejándolos de los propios de la experiencia humana, algo que refuerza los sentimientos negativos propios de la obra. Se trata de una estrategia sumamente interesante, y que sin embargo no es posible identificar mediante el análisis cuantitativo. Solo desde el estudio cualitativo propio del análisis literario es factible identificarlo y comprender su relevancia.

En relación con la segunda de estas áreas, la psicología de las emociones, el presente trabajo parte del marco conceptual desarrollado por Plutchik, y más concretamente de su conocida rueda de las ocho emociones básicas (Plutchik, 2009). El foco se ha puesto en la emoción dominante, que como se comprobará más adelante mediante el uso de diversas herramientas cuantitativas, es el miedo, y su relación con otras emociones también muy relevantes en la obra. De acuerdo a la teoría de Plutchik, la combinación de dos emociones primarias genera díadas, de modo que, por ejemplo, la mezcla de miedo y confianza genera sumisión, del mismo modo que la combinación de miedo y tristeza produce desesperación (véase la figura 1). Por esta razón resulta de interés analizar no solo las emociones primarias dominantes, sino también su interrelación, así como su evolución a lo largo de la obra. La implementación del modelo de Plutchik se ha articulado a través del lexicon de emociones NRC (Mohammad y Turney, 2013). Dicho lexicón es un listado de palabras en lengua inglesa (unigramas), de modo que cada una de ellas está asociada a una o varias de las emociones identificadas en la rueda de Plutchik. Por ejemplo, la palabra "abandoned" está asociada a sentimientos de tristeza, miedo e ira. Lógicamente, el análisis requiere del uso de herramientas computacionales, lo que nos lleva a la tercera área de trabajo, la minería de textos. En este sentido, el algoritmo programado para realizar los cálculos sigue el esquema habitual en modelos Bag-of-Words. Inicialmente se ha llevado a cabo el preprocesamiento de los datos, que implica ciertas modificaciones sobre todas las palabras que componen cada obra: transformación de letras mayúsculas a minúsculas, eliminación de signos de puntuación, eliminación de las denominadas stop words o palabras vacías (palabras que carecen de significado en sí mismas, pues modifican o acompañan a otras, como artículos, pronombres o preposiciones) y tokenización (división del texto en piezas más pequeñas o tokens, que en el caso de este trabajo son palabras). Posteriormente se ha empleado el lexicón NRC para asociar cada token a los correspondientes sentimientos. De este modo, según la frecuencia relativa de todas las palabras contenidas en una determinada obra, es posible calcular la importancia de cada una de las emociones. Se trata por tanto de un algoritmo de análisis de sentimientos (sentiment analysis) similar al que se emplea habitualmente en otros campos como el estudio de tweets o el análisis de discursos, aunque adaptado a las peculiaridades propias de una obra literaria. El algoritmo se ha programado íntegramente en $\mathrm{R}$ (R Core Team, 2013), un entorno orientado al análisis estadístico, empleando diversas librerías para elaborar el código: tidytext (Silge \& Robinson, 2016), ggplot2 (Wickham, 2016), fmsb (Nakazawa, 2018), tidyr (Wickham \& Henry, 2019), scales (Wickham, 2018), stringr (Wickham, 2019), dplyr (Wickham, François, Henry y Müller, 2020), y readtext (Benoit y Obeng, 2020). 
Figura 1. Principales sentimientos en The Call of Cthulhu de acuerdo a la rueda de las ocho emociones básicas de Plutchik

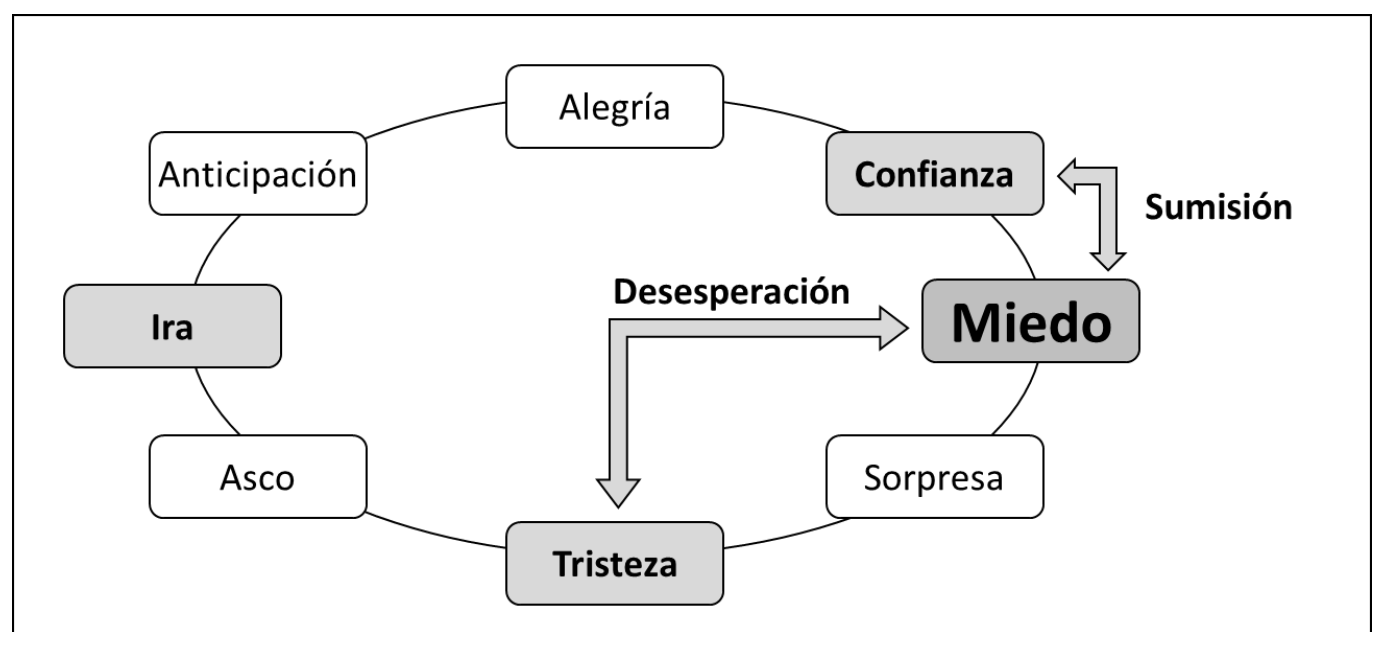

\section{THE CALL OF CTHULHU: ANÁLISIS DE SENTIMIENTOS}

The Call of Cthulhu es un relato corto estructurado en tres partes claramente diferenciadas, y que coinciden con los tres capítulos de la obra. En la primera, The Horror in Clay, Francis Wayland Thurston, el narrador de la historia, explica cómo, tras el fallecimiento de su tío-abuelo, el respetado académico Profesor Angell ${ }^{4}$, descubre diversos documentos en relación a un oscuro culto. Su investigación sobre este material le lleva a conocer la historia del Inspector Legrasse, que constituye la segunda parte del relato. En ella se describe como, en el curso de una investigación policial, Legrasse entra en contacto con una brutal secta que parece adorar a la criatura representada en un inquietante ídolo. Finalmente, en la tercera parte, se narra el descubrimiento accidental por parte de un marino noruego, Gustaf Johansen, de una extraña isla en la que entra en contacto con el ser representado en la estatua, Cthulhu.

Mediante el uso de herramientas de minería de textos se ha llevado a cabo un análisis de los sentimientos transmitidos por la obra en su conjunto (véase figura 2), y como puede observarse, las tres emociones dominantes son miedo, tristeza y confianza. En el eje vertical de la figura se muestra el peso relativo de cada una de las ocho emociones, siendo su suma el $100 \%$. De este modo, las emociones de miedo, tristeza y confianza tienen un peso relativo de entorno al $20 \%, 13 \%$ y $16 \%$ respectivamente. $\mathrm{O}$, dicho de otro modo, estos tres sentimientos suponen casi el 50\% de las emociones que transmite la obra. Si bien la relevancia de las dos primeras resulta esperable, dada la temática y estilo narrativo del autor, la importancia del sentimiento de confianza es menos evidente. Como analizaremos en detalle más adelante, se aprecia aquí un doble propósito por parte del autor: dotar de verosimilitud a un relato, que dados los extraordinarios hechos que describe resulta difícilmente creíble, y generar, en combinación con la emoción de miedo, un sentimiento de sumisión, clave en una obra que pretende destacar la insignificancia del ser humano ante fuerzas más allá de su comprensión.

\footnotetext{
${ }^{4}$ La presencia de este tipo de personajes es algo habitual en la obra de Lovecraft. Como señala Houellebecq (2019: 128) «in his stories the role of the victim is generally played by an Anglo-Saxon university professor who is refined, reserved, and well-educated».
} 
Figura 2. Peso relativo de las ocho emociones básicas en The Call of Cthulhu

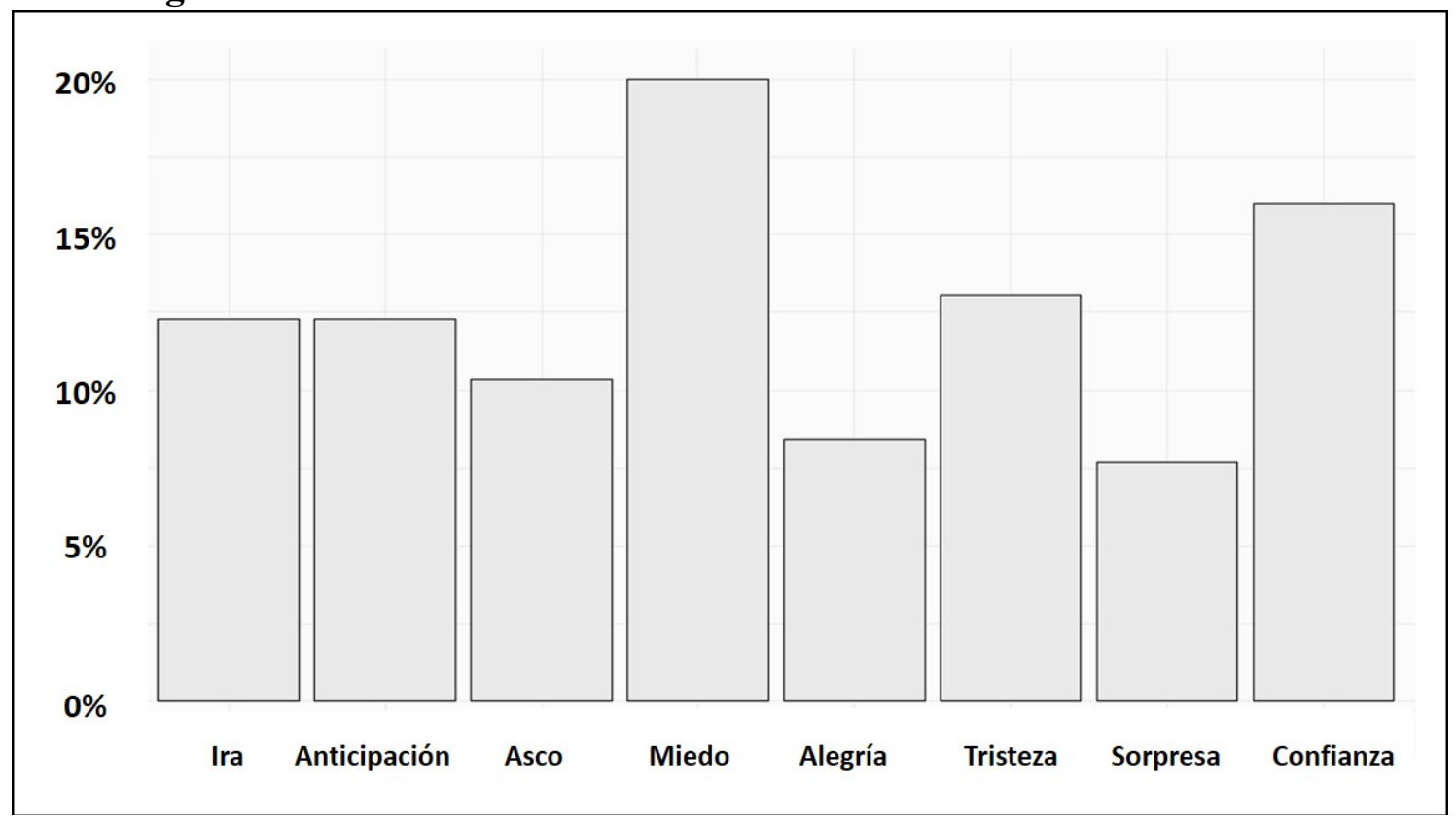

Si analizamos cómo estos sentimientos evolucionan a lo largo de la obra, distinguiendo entre cada una de sus partes (figura 3), observamos que la confianza va decreciendo, lo que resulta lógico, pues es en la primera parte en la que resulta más necesario establecer esa pátina de credibilidad. Del mismo modo, resulta interesante comprobar como la ira sufre un importante crecimiento hacia el final de la obra. Esto, como se analizará en detalle más adelante, está íntimamente ligado a la reacción final de enfrentamiento o huida.

Figura 3. Peso relativo de las ocho emociones básicas en las tres partes de The Call of Cthulhu

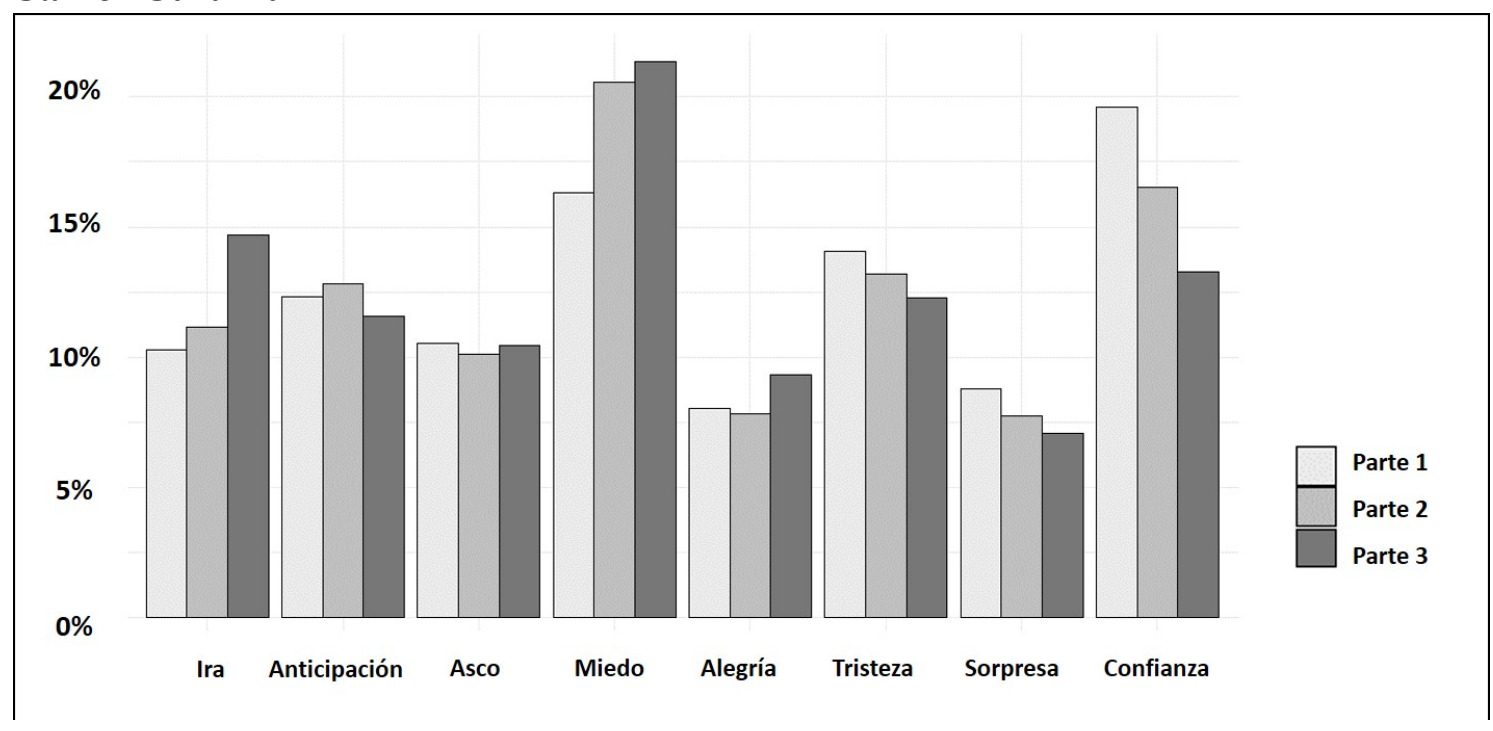

Entrando ya en el análisis detallado de cada una de estas emociones, comprobamos que, exactamente como cabría esperar, la atmósfera de tristeza es un elemento clave a lo largo de todo el relato pues la ficción de Lovecraft «focus on producing strong atmospheres of death, darkness and melancholy» (Norman, 2013: 6). Si 
bien no es la emoción dominante en el relato, sí resulta clave en su ambientación, y constituye un elemento central en el estilo narrativo de Lovecraft.

De hecho, la emoción dominante es el miedo, «the oldest and strongest emotion of mankind» (Lovecraft 2013[1935]: 1), así como otras emociones asociadas al mismo. En este sentido resulta clave destacar que el miedo que transmite no es de tipo físico, sino que se basa en «a certain atmosphere of breathless and unexplainable dread of outer, [...] a malign and particular suspension or defeat of those fixed laws of Nature [...]». (Lovecraft 2013[1935]: 2). Determinados elementos son utilizados sistemáticamente por Lovecraft para generar este efecto. En primer lugar, el arte, pues como señala Ralickas (2008: 314 ) existe una «intimate connection shared by art, the effect of cosmic horror, and the nihilistic, anti-humanist perspective of Lovecraft's fiction». De hecho, en la obra de Lovecraft los objetos étnicos, como estatuillas, ídolos o fetiches, «are usually indicators of things far worse than mere murder» (Hefner, 2014: 667-668). La minuciosa descripción que realiza del ídolo hallado por el Inspector Legrasse es un buen ejemplo de ello:

This thing [the idol], which seemed instinct with a fearsome and unnatural malignancy, was of a somewhat bloated corpulence, and squatted evilly on a rectangular block or pedestal covered with undecipherable characters. [...] The aspect of the whole was abnormally life-like, and the more subtly fearful because its source was so totally unknown. Its vast, awesome, and incalculable age was unmistakable. [...]. [The characters along the base of the idol] like the subject and material, belonged to something horribly remote and distinct from mankind as we know it.

(Lovecraft, 2017a[1928]: 41-42)

De hecho, en estas descripciones encontramos también otra de las señas de identidad en el estilo de Lovecraft, que enfatiza las limitaciones del lenguaje para describir los objetos, pues estos muestran a «seismic torsion with their own qualities» (Harman, 2012: 27). Este fracaso del lenguaje es, de hecho, «un descalabro más de la insignificante episteme humana» (Hernández Roura, 2019: 31).

En segundo lugar, la obra de Lovecraft contiene numerosas alusiones a sonidos, siempre caracterizados por su naturaleza desagradable, que representan el terror total de lo desconocido (van Elferen, 2016). Este elemento también está presente en The Call of Cthulhu, jugando un papel clave en la descripción que el Inspector Legrasse hace de su encuentro con la secta adoradora de Cthulhu.

Only poetry or madness could do justice to the noises heard by Legrasse's men as they ploughed on through the black morass toward the red glare and the muffled tom-toms. There are vocal qualities peculiar to men, and vocal qualities peculiar to beasts; and it is terrible to hear the one when the source should yield the other. Animal fury and orgiastic licence here whipped themselves to daemoniac heights by howls and squawking ecstasies that tore and reverberated through those nighted woods like pestilential tempests from the gulfs of hell

(Lovecraft, 2017a[1928]: 43)

Íntimamente ligados a estas alusiones sonoras encontramos los teratónimos de Lovecraft (Robinson, 2010), presentes en gran parte de su obra y abundantes en The Call of Cthulhu. De hecho la impronunciable frase «Cthulhu fhtagn», utilizada posteriormente por Lovecraft en otros relatos como The Shadow over Innsmouth (Lovecraft, 2017b[1936]) y que tanto ha permeado en el ámbito de la cultura popular, se menciona en repetidas ocasiones a lo largo del relato.

Por último, las matemáticas y en particular la geometría, son empleadas «to help build the mood for which he was striving» (Hull 2006: 10), describiendo estructuras 
anormales o dimensiones extrañas para crear una atmósfera intimidante. Dado el interés de Lovecraft en la ciencia, la relevancia de los trabajos de Einstein en el momento en que escribió su obra, y en tanto que la Relatividad General se sustenta en conceptos geométricos, no resulta extraño que «Einstein [...] runs through Lovecraft's fiction like an elusive thread» (Joshi, 2014a), a pesar de que seguramente nunca leyó los trabajos originales del afamado físico, sino que probablemente accedió a sus ideas desde fuentes populares (Joshi, 2016: 23). Como señala Ingwersen (2014: 57), los conceptos de espacio curvo y dimensiones insondables son motivos recurrentes en los mundos de Lovecraft, lo que este autor llama «the horror of geometry», cuyas influencias debemos buscar en los desarrollos científicos del momento, incluyendo las teorías de Einstein y, por supuesto, la geometría de Riemann en la que se fundamenta la Relatividad General. El uso de este recurso es especialmente abundante en la tercera parte del relato, que basa gran parte de la descripción de R'lyeh, la morada de Cthulhu, en destacar la imposibilidad del lenguaje para expresar adecuadamente las características geométricas de su estructura.

He had said that the geometry of the dream-place he saw was abnormal, non-Euclidean, and loathsomely redolent of spheres and dimensions apart from ours. [...]. The very sun of heaven seemed distorted when viewed through the polarising miasma welling out from this sea-soaked perversion, and twisted menace and suspense lurked leeringly in those crazily elusive angles of carven rock where a second glance shewed concavity after the first shewed convexity

(Lovecraft, 2017a[1928]: 51)

Donovan slid or somehow propelled himself down or along the jamb and rejoined his fellows, and everyone watched the queer recession of the monstrously carven portal. In this phantasy of prismatic distortion it moved anomalously in a diagonal way, so that all the rules of matter and perspective seemed upset. [...]. Parker slipped as the other three were plunging frenziedly over endless vistas of green-crusted rock to the boat, and Johansen swears he was swallowed up by an angle of masonry which shouldn't have been there; an angle which was acute, but behaved as if it were obtuse

(Lovecraft, 2017a[1928]: 52)

En resumen, el miedo, como emoción dominante del relato, se articula en torno a distintos elementos como el arte, los sonidos y las matemáticas, que buscan un efecto muy diferente al que provoca el mero miedo físico basado en el instinto animal, enfatizando en todos los casos las limitaciones del lenguaje para describir adecuadamente dichos elementos.

Por otra parte, la segunda emoción más importante en The Call of Cthulhu es la confianza (véanse las figuras 2 y 3 ). Joshi (2014b) señala que «the use of many types of documents - letters, diaries, newspaper articles, [...] — serves to 'distance' the narrative and subtilise it». Adicionalmente esta estrategia narrativa sirve a un propósito adicional, que es dotar de verosimilitud al relato, sustentando los hechos en un soporte físico que confiere credibilidad a los hechos descritos. Como Lovecraft mencionó en su correspondencia con Clark Ashton Smith «No weird story can truly produce terror unless it is devised with all the care \& verisimilitude of an actual hoax» (H. P. Lovecraft a Clark Ashton Smith 17 de Octubre de 1930. Citado en Joshi, 2014d). Sustentar la historia en una cronología de informaciones meticulosamente recopiladas de fuentes teóricamente fiables, tales como periódicos o las notas tomadas por un erudito profesor de universidad, es una excelente manera de dotar de credibilidad al relato, que, dadas sus extraordinarias características, sería muy difícil de aceptar. De hecho, las credenciales de las voces individuales (Profesor Angell, Inspector Legrasse y el propio Thurston) son cuidadosamente documentadas, destacando su credibilidad y objetividad (Anderson, 1992: 42-43). Como apunta Joshi (2015: 59-60), un aspecto clave de The Call of Cthulhu 
es precisamente su uso de la verosimilitud científica para superar el escepticismo del lector respecto a los extraños acontecimientos que se relatan. Realmente se trata de un esquema habitual en toda la obra de Lovecraft en la que «an accumulation of place-based evidence, both folkloric and scientific, leads [...] to a realization of the illusory nature of truth and the unknowability of the cosmos» (Evans, 2005: 123) ${ }^{5}$. Como señala Sorensen (2010: 502) «Lovecraft uses techniques from ethnography and antiquarianism to produce both a model of culture and a fantastic universe that draws its claims to verisimilitude by means of a strategic practice of citation». De este modo, aportando datos, fechas y detalles sustentados en diversos soportes, proporciona una sensación de verosimilitud que difumina las fronteras entre fantasía y realidad. ${ }^{6}$ Expresado según las propias palabras de Lovecraft:

One cannot [...] present an account of impossible, improbable, or inconceivable phenomena as a commonplace narrative of objective acts and conventional emotions. Inconceivable events and conditions have a special handicap to overcome, and this can be accomplished only through the maintenance of a careful realism in every phase of the story except that touching on the one given marvel

(Lovecraft, 2009[1937]: 1)

Por último, debemos mencionar que en un reciente trabajo de Hernández Roura (2019), se lleva a cabo un minucioso análisis de los distintos recursos retóricos utilizados por Lovecraft para convencer de la verosimilitud de lo imposible, por lo que no insistiremos más en este particular, remitiendo a dicho trabajo al lector interesado en profundizar en el mismo.

Sin embargo, adicionalmente a lo expuesto, la emoción de confianza juega otro papel crítico en The Call of Cthulhu, en tanto que, como se ha comentado previamente, la combinación de miedo y confianza genera un sentimiento clave en la obra: sumisión. MacCormack (2010) señala que «Lovecraft's cosmic horror works are obsessed with the idea of relations that inevitably structure and underpin human existence but that remain unknown to the human». Aceptar esta terrible realidad, implica necesariamente la sumisión ante fuerzas que escapan de la compresión humana. Muy ligado a este sentimiento está el de desesperación, que surge como combinación de miedo y tristeza, una desesperación basada parcialmente en la idea de que, por encima de la perspectiva nihilista, puede haber un más allá, pero es algo habitado por el mal, incluso peor que el «meaningless world of material reality» (Hanegraaff, 2007: 89). Como señala Joshi (2014b) «for in fact the true climax of the tale is not the emergence of Cthulhu (who in any case vanishes after a few moments), but the implication of the narrative - the implication that hideous, vastly powerful entities lurk just behind our consciousness». Ambas emociones, sumisión y desesperación, están muy presentes en The Call of Cthulhu, y quedan claramente reflejadas en las últimas páginas del relato, en las que el narrador Francis Wayland Thurston, reflexiona sobre las implicaciones de sus descubrimientos:

\footnotetext{
${ }^{5}$ Resulta de interés mencionar aquí la reflexión de Weik von Mossner (2017: 163. Citada en Ulstein, 2019), quien apunta que "an overload of negative emotions [such as fear, sadness or anger] might either lead to debilitating pessimism or to various forms of denial". Reforzando la credibilidad del relato, ciertamente repleto de emociones negativas, Lovecraft evita esa evasión del lector mediante la negación de los hechos. ${ }^{6}$ Esta peculiaridad, presente en muchas de las obras de Lovecraft es, desde nuestro punto de vista, uno de los factores clave que han contribuido al notable malentendido con el Necronomicón, un texto ficticio inventado por Lovecraft dentro de su mitología y que incluso hoy en día, muchos consideran un libro real (véase Harms \& Gonce, 2003). A esto hemos de añadir que, a través de técnicas intertextuales como la alusión, el Necronomicón «has gained materiality» (Lippert, 2012: 41).
} 
Death would be a boon if only it could blot out the memories. [...]

I have looked upon all that the universe has to hold of horror, and even the skies of spring and the flowers of summer must ever afterward be poison to me. But I do not think my life will be long. As my uncle went, as poor Johansen went, so I shall go. I know too much, and the cult still lives.

Cthulhu still lives, too, I suppose, again in that chasm of stone which has shielded him since the sun was young. [...]. Loathsomeness waits and dreams in the deep, and decay spreads over the tottering cities of men

(Lovecraft, 2017a[1928]: 53-54)

Para terminar con el análisis, hemos de prestar atención a una emoción que apenas cobra relevancia hacia el final del relato: la ira. Como se observa en la figura 3, este sentimiento se mantiene en niveles relativamente bajos hasta la tercera parte, el encuentro de Gustaf Johansen con el propio Cthulhu, momento en el que adquiere una importancia solo superada por el miedo. Sin embargo, para comprender plenamente su sentido, hemos de analizar otro aspecto de la obra que resulta crítico a fin de obtener una imagen final del estilo narrativo de Lovecraft: un súbito cambio en el cronotopo. Partiendo de la definición original de Bakhtin (1990) podemos definir el cronotopo como la conexión entre los elementos temporales y espaciales expresados artísticamente en la literatura. Espacio y tiempo juegan un papel muy relevante en la obra de Lovecraft. Su estilo narrativo se apuntala en un realismo topográfico y documental minucioso, que combina magistralmente geografía y bibliografía fantásticas y reales (Gómez Alonso, 2012), y en el que espacios y lugares son esenciales (Kneale, 2016). Por otra parte, el tiempo en Lovecraft es peculiar, en tanto que al tiempo de la narración se superpone la idea de un tiempo a escala universal, es decir, un tiempo ante el cual «the entirety of human history does not measure, [...] a scale which the human mind cannot conceive» (Smith, 2011: 837). La unión de ambos elementos configura el cronotopo que, si bien en la primera y segunda parte de la obra resulta desconcertante por la extraña superposición del tiempo de narración y el tiempo universal, se mantiene dentro de un esquema relativamente próximo a la realidad ordinaria. Sin embargo, en la tercera parte, a medida que se aproxima el clímax, observamos un cambio radical que se materializa en el momento en que Johansen llega a la extraña isla surgida de las profundidades del océano Pacífico. Por una parte, resulta significativo el recurso de la isla, utilizado por Lovecraft también en otros relatos como Dagon (Lovecraft, 2017e[1919]), pues como señala Kneale (2017), existe una relación entre las islas literarias y los temas de transformación, que permite el encuentro con la alteridad. Adicionalmente, la extraña naturaleza de la isla, basada en una geometría no euclídea imposible de comprender por el marinero noruego, supone un cambio radical con respecto al cronotopo, mucho más próximo a la experiencia humana, de las dos partes anteriores. Es en esta isla, que conforma un cronotopo netamente diferente al del resto del relato, donde efectivamente se produce la transformación a que hace referencia Kneale. Con un súbito aumento del sentimiento de ira, Johansen reacciona ante la amenaza, y en lugar de quedar atenazado por el terror, ataca a Cthulhu envistiéndolo con su barco, logrando así una fútil victoria. He aquí la razón para la peculiar evolución del sentimiento de ira en la última parte del libro: un intento final de rebelión, enmarcado en un cronotopo ligado precisamente a la transformación.

En resumen, Lovecraft emplea un patrón muy concreto en The Call of Cthulhu articulado en torno a tres ejes: la creación de una atmósfera vertebrada en base a las emociones de miedo, confianza y tristeza, en ese orden de importancia; unos mayores niveles de confianza en las primeras partes de la obra, a fin de establecer una base de credibilidad para los extraordinarios hechos narrados en el clímax; $\mathrm{y}$, por último, un giro 
final, ligado a un aumento del sentimiento de ira, en el que la reacción de quien entra en contacto con la monstruosa criatura le confiere una fútil victoria.

\section{UN PATRÓN RECURRENTE}

Resulta de interés analizar hasta qué punto este patrón aparece en otras obras de Lovecraft, para lo que se han elegido tres de sus trabajos icónicos, The Shunned House (Lovecraft, 2017c[1928]), The Case of Charles Dexter Ward (Lovecraft, 2017d[1941]) y The Mound (Lovecraft, 2020). Todas ellas comparten con The Call of Cthulhu el uso de lo que Joshi (2014b) denomina «the direct narrative within a narrative, for in that tale the narrator himself has pieced together some of the information representing the subsidiary narratives». Como puede comprobarse, existe una notable homogeneidad en las cuatro obras consideradas globalmente (véase la figura 4). En todos los casos miedo, confianza y tristeza son las emociones dominantes, y, salvo en el caso de The Shunned House, su relevancia sigue exactamente ese orden. Esto confirma la hipótesis respecto al primer eje que se mencionaba en el apartado anterior.

Figura 4. Peso relativo de las ocho emociones básicas en los cuatros relatos analizados

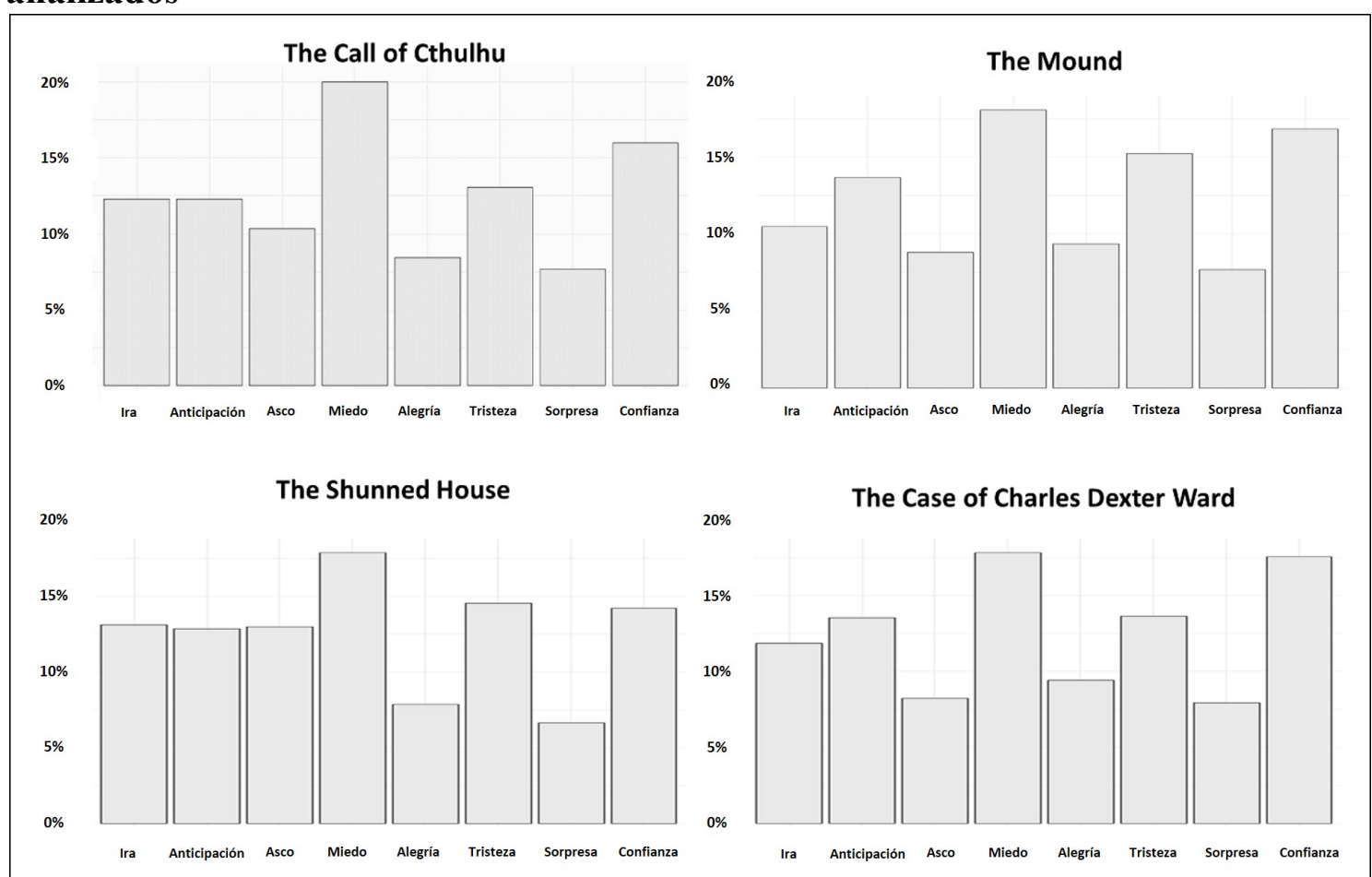

Para evaluar hasta qué punto se verifican las otras dos hipótesis planteadas, es necesario analizar la evolución de los sentimientos a lo largo de las distintas obras, organizadas de acuerdo a su contenido. Así, por ejemplo, los siete capítulos de The Mound se han estructurado en tres partes: la introducción del relato (capítulos 1 y 2), la historia de Pánfilo de Zamacona (capítulos 3, 4, 5 y 6), y el clímax de la novela, con la entrada del narrador en el montículo (capítulo 7). En la figura 5 se muestran los resultados del análisis. 
Figura 5. Peso relativo de las ocho emociones básicas en cada parte de los cuatro relatos analizados, indicando entre paréntesis qué capítulos incluye

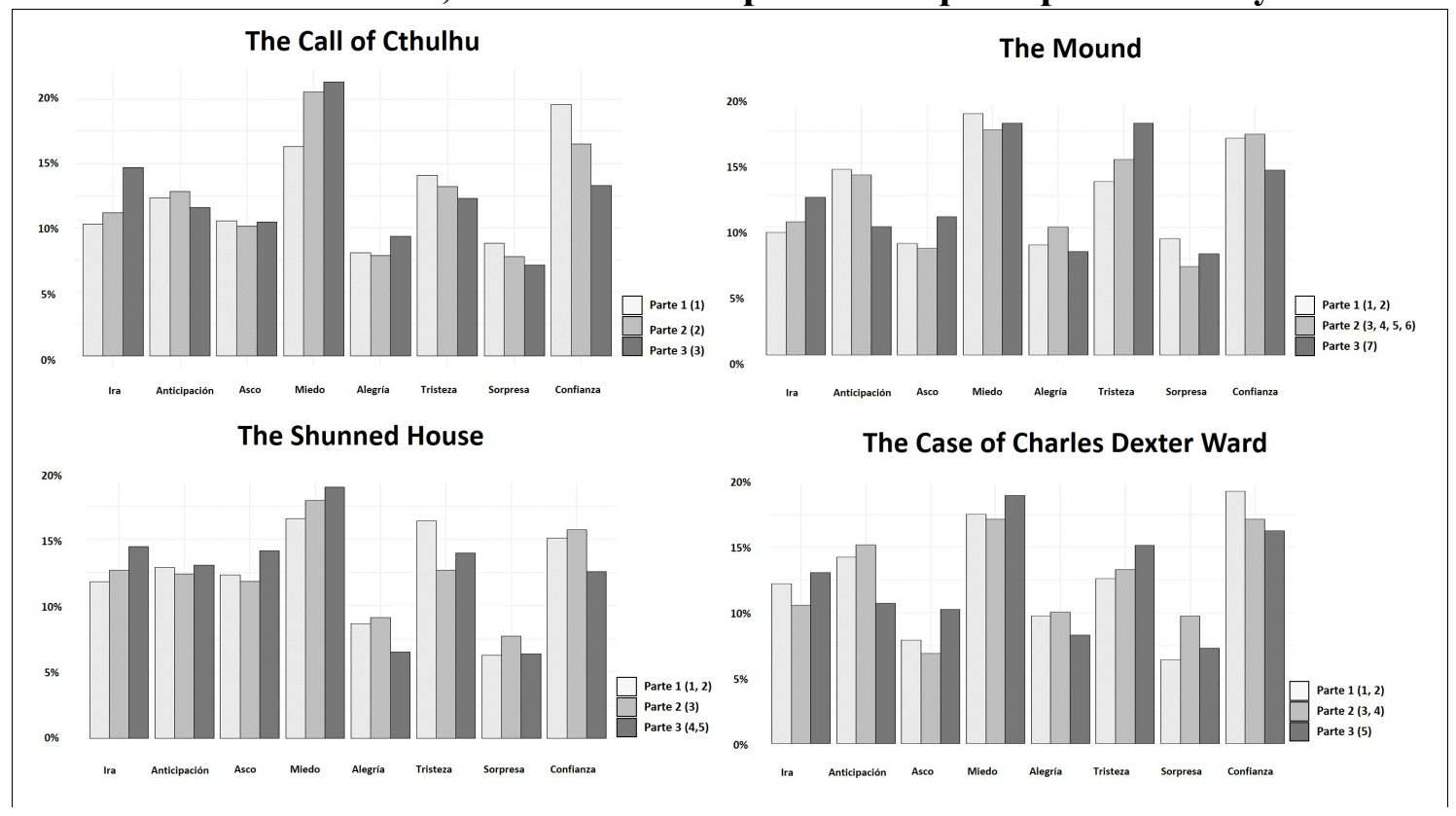

En relación con unos mayores niveles de confianza al principio de la obra, el segundo eje que se mencionaba en el apartado anterior, observamos que efectivamente parece confirmarse, pues los niveles de este sentimiento son mínimos justo al final de cada relato. Debemos indicar que, si se replica este análisis estructurando las obras por capítulos, las conclusiones son idénticas con una excepción: en The Mound encontramos un patrón anómalo en los tres primeros, con niveles de confianza más bajos de lo esperado. Resulta interesante que esto suceda precisamente en el único de los cuatro relatos sobre el que existen ciertas dudas en cuanto a la autoría completa de Lovecraft. La obra fue escrita por Lovecraft para Zealia Bishop, actuando como escritor fantasma, y no queda claro cuál fue el nivel de participación de Bishop u otros en el manuscrito final. De hecho, Zealia Bishop afirma en sus memorias que Frank Belknap Long ayudó a revisar el manuscrito, con lo que estaríamos hablando incluso de un tercer interviniente (Joshi, 2014e). Joshi (2014e) afirma que el texto fue enteramente escrito por Lovecraft, en base a la idea inicial aportada por la escritora, aunque existe una confusa historia con respecto a los cambios, correcciones y rectificaciones del manuscrito. Lo cierto es que esta anomalía, bajos niveles de confianza en los tres primeros capítulos, sería compatible con que la participación de Bishop o Long hubiese sido mayor de lo que Joshi propone, especialmente en la primera parte de la obra. Aquí se abre una interesante línea de investigación, ya que las modernas técnicas de minería de textos permiten analizar cuantitativamente el problema de la atribución autoral, por lo que en futuros trabajos se plantea explorar este particular. Sin embargo, y al margen de este matiz en The Mound, el análisis cuantitativo que se ha llevado a cabo mediante herramientas de minería de datos confirma que en las cuatro obras estudiadas «los sucesos narrados se encadenan con la finalidad de preparar al lector para atestiguar la transgresión [la irrupción de un fenómeno imposible]» (Hernández Roura, 2019: 23).

Del mismo modo, observamos una evolución creciente del sentimiento de ira, que alcanza su máximo en el clímax de la obra. No obstante, hemos de indicar que únicamente en dos de ellas se produce un cambio en el cronotopo para reforzar el clímax: The Call of Cthulhu, ya analizada en el apartado anterior y The Shunned House. En esta última, si bien a lo largo de la novela ya encontramos puntualmente algunos elementos propios del 
cronotopo de la casa encantada, es justo al final cuando la capacidad de la casa «to act as a physical conduit for the paradoxical return of these troubled pasts to an often equally troubled present» (Prosser, 2015: 6) se presenta en toda su magnitud. En este sentido resulta muy revelador uno de los pasajes finales de la obra, en la que se manifiestan los espíritus de los distintos moradores de la casa.

I saw the features of the Harris line, masculine and feminine, adult and infantile, and other features old and young, coarse and refined, familiar and unfamiliar. For a second there flashed a degraded counterfeit of a miniature of poor mad Rhoby Harris that I had seen in the School of Design Museum, and another time I thought I caught the raw-boned image of Mercy Dexter as I recalled her from a painting in Carrington Harris's house

(Lovecraft, 2017c[1928]: 617-618)

\section{CONCLUSIONES}

A lo largo de este trabajo se ha analizado la presencia de un patrón muy específico tanto en The Call of Cthulhu como en otras de las obras icónicas de Lovecraft. Combinando el análisis literario con técnicas de minería de textos, y más concretamente de sentiment analysis, se ha podido cuantificar dicho patrón, que se articula en torno a tres ejes. El primero de ellos es la creación de una atmósfera vertebrada en base a las emociones de miedo, confianza y tristeza. Se trata de una conclusión que ya había sido tratada previamente por diversos académicos, tal y como se ha analizado a lo largo del artículo, por lo que hasta aquí no resulta especialmente novedosa. Sin embargo, en este trabajo se ha podido cuantificar el peso de cada una de estas emociones, llegando a la conclusión de que su importancia relativa es sorprendentemente homogénea en los cuatro relatos analizados: miedo, confianza y tristeza siguen precisamente este orden de importancia. Este resultado es de interés por el hecho de repetirse en las obras estudiadas, y en futuras investigaciones se podría tratar de verificar si efectivamente es la norma en toda la producción literaria de Lovecraft.

En segundo lugar, se observan unos mayores niveles de confianza en las primeras partes de la obra, a fin de establecer una base de credibilidad para los extraordinarios hechos narrados en el clímax. Como señala Hernández Roura (2019: 23) «los sucesos narrados se encadenan con la finalidad de preparar al lector para atestiguar la transgresión [la irrupción de un fenómeno imposible]», y en este trabajo se ha podido confirmar cuantitativamente esta evolución, verificando su presencia en las cuatro obras analizadas.

Por último, el tercer eje analizado en este trabajo es el giro final, ligado a un aumento del sentimiento de ira, una reacción de quien entra en contacto con las monstruosas criaturas, confiriéndole una victoria que realmente resulta inútil a largo plazo, como, en muchos casos, el propio narrador se encarga de explicitar en los últimos párrafos del texto. Esta reacción última puede ir acompañada de un cambio en el cronotopo, como sucede en The Call of Cthulhu y en The Shunned house, enfatizando así la idea de transformación. Siendo la ira la emoción opuesta al miedo de acuerdo a la teoría de Plutchik, cabe preguntarse por las razones que llevaron a Lovecraft incorporar sistemáticamente este patrón. ¿Tiene esto sentido en unos relatos que, precisamente, inciden en la insignificancia de la raza humana y la futilidad de todas sus acciones? ¿Qué sentido tiene rebelarse ante lo inevitable? Quizá estamos ante un intento de reforzar esta idea, explicitando que ninguna victoria será duradera, pues una vez se accede a los misterios que deberían permanecer ocultos, la muerte, o algo peor, es el único destino posible. O expresándolo en palabras de Francis Wayland Thurston, el narrador de The Call of Cthulhu, «[...] I do not think my life will be long. As my uncle went, as poor Johansen went, so I shall go» (Lovecraft, 2017a[1928]: 53). 


\section{REFERENCIAS BIBLIOGRÁFICAS}

Anderson, James A. 1992. Out of the Shadows: A Structuralist Approach to Understanding the Fiction of H. P. Lovecraft. Tesis doctoral, Universidad de Rhode Island. Disponible en https://digitalcommons.uri.edu/oa_diss/696 [último acceso 9/7/2020]

Bakhtin, Mikhail M. 1990. «Forms of Time and of the Chronotope in the Novel: Notes toward a Historical Poetics». En Michael Holquist (ed.), The Dialogic Imagination: Four Essays. Austin: University of Texas Press, 84-258.

Benoit, Kenneth, y Adam Obeng. 2020. readtext: Import and Handling for Plain and Formatted Text Files. $\mathrm{R}$ package version 0.76. https:/CRAN.Rproject.org/package $=$ readtext

De Camp, L. Sprague. 2011. Lovecraft: A Biography. Gateway, Edición de Kindle.

Evans, Timothy H. 2005. «A last defense against the dark: Folklore, horror, and the uses of tradition in the works of HP Lovecraft». Journal of folklore research, 42(1): 99-135.

Gómez-Alonso, Modesto Manuel. 2012. «HP Lovecraft: creencia estética y asentimiento intelectual». Taula: quaderns de pensament, 44: 141-152.

Hanegraaff, Wouter J. 2007. «Fiction in the desert of the real: Lovecraft's Cthulhu Mythos». Aries, 7(1): 85-109.

Harman, Graham. 2010. «On the horror of phenomenology: Lovecraft and Husserl». Collapse IV: Philosophical Research and Development. Disponible en: https://www.urbanomic.com/book/collapse-4/ [ultimo acceso: 10/07/2020]

Harman, Graham. 2012. Weird realism: Lovecraft and philosophy. Winchester: Zero Books.

Harms, Daniel, y John Wisdom Gonce. 2003. The Necronomicon files: The truth behind Lovecraft's legend. York Beach: Weiser Books.

Hefner, Brooks E. 2014. "Weird Investigations and Nativist Semiotics in HP Lovecraft and Dashiell Hammett». Modern Fiction Studies, 60(4): 651-676. https://doi.org/10.1353/mfs.2014.0054

Hernández Roura, Sergio Armando. 2019. «Rhetorics and Cosmicism in HP Lovecraft». Brumal. Revista de investigación sobre lo Fantástico, 7(1): 15-34. https://doi.org/10.5565/rev/brumal.503

Houellebecq, Michel. 2019. H. P. Lovecraft: Against the World, Against Life. Cernunnos. Kindle Edition. Traducción de D. Khazeni.

Hull, Thomas. 2006. «HP Lovecraft: A Horror in Higher Dimensions». Math Horizons, 13(3): 10-12. https://doi.org/10.1080/10724117.2006.11974625

Ingwersen, Moritz. 2014. «Monstrous Geometries in the fiction of Hp Lovecraft». En Craig Douglas y Rosalea Monacella (eds.), Places and Spaces of Monstrosity. Oxford: Interdisciplinary Press, 57-67.

Joshi, Sunand Tryambak. 2007. «The Cthulhu Mythos». En Sunand Tryambak Joshi (ed.), The Cthulhu Mythos. Icons of Horror and the Supernatural: An Encyclopedia of Our Worst Nightmares, Vol. 1. Westport: Greenwood Press, 97128.

Joshi, Sunand Tryambak. 2014a. «Topical References in Lovecraft». En Sunand Tryambak Joshi (ed.), Lovecraft and a World in Transition: Collected Essays on H. P. Lovecraft. Hippocampus Press, edición de Kindle.

Joshi, Sunand Tryambak. 2014b. «The Structure of Lovecraft's Longer Narratives». En Sunand Tryambak Joshi (ed.), Lovecraft and a World in Transition: Collected Essays on H. P. Lovecraft. Hippocampus Press, edición de Kindle. 
Joshi, Sunand Tryambak. 2014c. «H. P. Lovecraft: The Fiction of Materialism». En Sunand Tryambak Joshi (ed.), Lovecraft and a World in Transition: Collected Essays on H. P. Lovecraft. Hippocampus Press, edición de Kindle.

Joshi, Sunand Tryambak. 2014d. «History of the Necronomicon». En Sunand Tryambak Joshi (ed.), Lovecraft and a World in Transition: Collected Essays on H. P. Lovecraft, Hippocampus Press. edición de Kindle.

Joshi, Sunand Tryambak. 2014e. «Who Wrote 'The Mound'?». En Sunand Tryambak Joshi (ed.), Lovecraft and a World in Transition: Collected Essays on H. P. Lovecraft. Hippocampus Press, edición de Kindle.

Joshi, Sunand Tryambak. 2015. The Rise, Fall, and Rise of the Cthulhu Mythos. Nueva York: Hippocampus Press.

Joshi, Sunand Tryambak. 2016. HP Lovecraft: The Decline of the West. Cabin John: Wildside Press LLC.

Joshi, Sunand Tryambak. 2017. «Literary geographies of possession, separation, and transformation». En Robert T. Tally Jr. (ed.), The Routledge Handbook of literature and space. Londres: Taylor \& Francis.

Kneale, James. 2006. «From beyond: HP Lovecraft and the place of horror». Cultural geographies, 13(1): 106-126. https://doi.org/10.1191/1474474005eu353oa

Lippert, Conny. 2012. «Lovecraft's Grimoires: Intertextuality and the Necronomicon», Working with English: Medieval and Modern Language, Literature and Drama, 8: 41-50.

Lovecraft, Howard Phillips. 2009. «Notes on writing weird fiction». En Donovan K. Loucks (ed.), The HP Lovecraft Archive. Primera publicación en 1937. Disponible en https://hplovecraft.com/ [último acceso 15/7/2020].

Lovecraft, Howard Phillips. 2013. «Supernatural horror in literature», Abergele: The Palingenesis Project (Wermod and Wermod Publishing Group). Primera publicación en Abril de 1935.

Lovecraft, Howard Phillips. 2017a. «The call of Cthulhu». En The Complete Fiction of H. P. Lovecraft. Oxford: Benediction Classics. Primera publicación en Weird Tales, 11(2): 159-178, Febrero 1928.

Lovecraft, Howard Phillips. 2017b. «The shadow over Innsmouth». En The Complete Fiction of H. P. Lovecraft. Oxford: Benediction Classics. Primera publicación en The Shadow over Innsmouth. Everett, PA: Visionary Publishing Co., 13-158, 1936.

Lovecraft, Howard Phillips. 2017c. «The shunned house». En The Complete Fiction of H. P. Lovecraft. Oxford: Benediction Classics. Primera publicación en The Shunned House, Athol, MA: The Recluse Press, 9-59, 1928,

Lovecraft, Howard Phillips. 2017d. "The case of Charles Dexter Ward». En The Complete Fiction of H. P. Lovecraft. Benediction Classics, Oxford. Primera publicación en Weird Tales, 35(9 y 10): 8-40 / 84-121, Mayo/Julio 1941.

Lovecraft, Howard Phillips. 2017e. «Dagon». En The Complete Fiction of H. P. Lovecraft. Benediction Classics, Oxford. Primera publicación en The Vagrant, Noviembre 1919.

Lovecraft, Howard Phillips. 2020. «The mound». En Donovan K. Loucks (ed.), The HP Lovecraft Archive. Primera publicación en 1937. Disponible en https://hplovecraft.com/ [último acceso 1/7/2020].

Mariconda, Steven J. 1991. «Lovecraft's Cosmic Imagery». En David E. Schultz y Sunand Tryambak Joshi (eds.), An Epicure in the Terrible: A Centennial Anthology of Essays in Honor of HP Lovecraft. Cranbury: Fairleigh Dickinson Univ Press, 188-198. 
MacCormack, Patricia. 2010. «Lovecraft through Deleuzio-Guattarian Gates». Postmodern Culture, 20(2). Disponible en: http://www.pomoculture.org/2013/09/03/lovecraft-through-deleuzioguattarian-gates/ [último acceso: 10/7/2020]

Menegaldo, Gilles. 2019. «H. P. Lovecraft on screen, a challenge for filmmakers (allusions, transpositions, rewritings)». Brumal. Revista de investigación sobre lo Fantástico, 7(1): 55-79. https://doi.org/10.5565/rev/brumal.591

Mohammad, Saif y Peter Turney. 2013. Nrc emotion lexicon. Technical report, NRC Technical Report. http://saifmohammad.com/WebPages/NRC-EmotionLexicon.htm

Nakazawa, Minato. 2018. fmsb: Functions for Medical Statistics Book with some Demographic Data, $\mathrm{R}$ package version 0.6.3. https://CRAN.Rproject.org $/$ package $=$ fmsb

Norman, Joseph. 2013. «Sounds Which Filled Me with an Indefinable Dread: The Cthulhu Mythopoeia of HP Lovecraft in Extreme Metal». En Davis Simmons (ed.), New Critical Essays on HP Lovecraft. Nueva York: Palgrave Macmillan, 193-208.

Peregrina Castaños, Mikel. 2019. «Ritos arcanos, poderes ocultos y horrores intemporales: la presencia del imaginario lovecraftiano en el metal extremo español reciente». Brumal. Revista de investigación sobre lo Fantástico, 7(1): 81-105. https://doi.org/10.5565/rev/brumal.545

Plutchik, Robert. 2009. «Emotions: A general psychoevolutionary theory». En Klaus R. Scherer y Paul Ekman (eds.), Approaches to emotion. Hilsdale: Psychology Press, 197-219.

Prosser, Ashleigh. 2015. «No Place Like Home: The Chronotope of the Haunted House in Peter Ackroyd's The House of Doctor Dee». Aeternum: The Journal of Contemporary Gothic Studies, 2(1): 1-19.

$\mathrm{R}$ Core Team. 2013. $R$ : A language and environment for statistical computing, $\mathrm{R}$ Foundation for Statistical Computing. URL: http://www.R-project.org/

Ralickas, Vivian. 2008. «Art, Cosmic Horror, and the Fetishizing Gaze in the Fiction of HP Lovecraft». Journal of the Fantastic in the Arts, 19(3): 297-316.

Robinson, Christopher L. 2010. «Teratonymy: The Weird and Monstrous Names of HP Lovecraft». Names, 58(3): 127-138. https://doi.org/10.1179/002777310X12759861710420

Silge, Julia, y David Robinson. 2016. «tidytext: Text Mining and Analysis Using Tidy Data Principles in R». Journal of Open Source Software, 1(3): 37. http://doi.org/doi:10.21105/joss.00037

Smith, Don G. 2006. HP Lovecraft in popular culture: The works and their adaptations in film, television, comics, music and games. Jefferson: McFarland.

Smith, Philip. 2011. «Re-visioning Romantic-Era Gothicism: An Introduction to Key Works and Themes in the Study of HP Lovecraft». Literature Compass, 8(11): 830-839. https://doi.org/10.1111/j.1741-4113.2011.00838.x

Sorensen, Leif. 2010. «A weird modernist archive: Pulp fiction, pseudobiblia, HP Lovecraft». Modernism/modernity, 17(3): 501-522. http://doi.org/10.1353/mod.2010.0007

Steadman, John L. 2015. HP Lovecraft and the Black Magickal Tradition: The Master of Horror's Influence on Modern Occultism. San Francisco: Weiser Books.

Stableford, Brian. 2007. «The Cosmic Horror». En Sunand Tryambak Joshi (ed.), The Cthulhu Mythos. Icons of Horror and the Supernatural: An Encyclopedia of Our Worst Nightmares, Vol. 1. Westport: Greenwood Press, 65-96. 
Ulstein, Gry. 2019. «Age of Lovecraft'? Anthropocene Monsters in (New) Weird Narrative». Nordlit, 42: 47-66. https://doi.org/10.7557/13.5004.

Van Elferen, Isabella. 2016. «Hyper-cacophony: Lovecraft, speculative realism, and sonic materialism». En Carl H. Sederholm y Jeffrey Andrew Weinstock (eds.), The Age of Lovecraft. Minneapolis: University of Minnesota Press, 79-96.

Weik VON Mossner, Alexa. 2017. Affective Ecologies: Empathy, Emotion, and Environmental Narrative. Columbus: Ohio State University Press.

Wickham, Hadley. 2016. ggplot2: Elegant Graphics for Data Analysis. Nueva York: Springer-Verlag.

Wickham, Hadley. 2018. scales: Scale Functions for Visualization. R package version 1.0.0. https://CRAN.R-project.org/package $=$ scales

Wickham, Hadley. 2019. stringr: Simple, Consistent Wrappers for Common String Operations. R package version 1.4.0. https://CRAN.Rproject.org/package $=$ stringr

Wickham, Hadley y Lionel Henry. 2019. tidyr: Tidy Messy Data. R package version 1.0.0. https://CRAN.R-project.org/package=tidyr

Wickham, Hadley, Romain François, Lionel Henry, y Kirill Müller. 2020. dplyr: A Grammar of Data Manipulation. R package version 0.8.5. https://CRAN.Rproject.org/package $=$ dplyr 